\title{
Sham neurosurgery in patients with Parkinson's disease: is it morally acceptable?
}

Wim Dekkers and Gerard Boer University Medical Centre, Nijmegen, the Netherlands and Netherlands Institute for Brain Research, Amsterdam, the Netherlands

\begin{abstract}
For a few decades, patients with Parkinson's disease (PD) have been treated with intracerebral transplantations of fetal mesencephalic tissue. The results of open trials have been variable. Double blind, placebo-controlled studies have recently been started in order to further investigate the efficacy of this new medical technique. In this paper we challenge the need for sham surgery in neurotransplantation research on $P D$ patients. Considerations regarding the research subjects' informed consent, therapeutic misconception, the integrity of the human body, and the assessment of risks and benefits argue against sham surgery for patients with PD. Moreover, there is an alternative, less harmful mode of research that can provide the same or comparable scientific evidence. A plea is made for intrapatient research based on quantitative measurements of the patient's pre-and post-operative condition combined with similar research on a reference group of patients who have received the standard treatment.

(Fournal of Medical Ethics 2001;27:151-156)
\end{abstract}

Keywords: Neural grafting; Parkinson's disease; research ethics; sham surgery

\section{Introduction}

For a few decades, the possibility of transplanting human body parts has become increasingly important in biomedical research and in the treatment of patients. ${ }^{1}$ One of the promising developments in this field is embryonic and fetal tissue transplantation (EFTT). The intracerebral transplantation of fetal tissue was given its first clinical trials in an attempt to treat Parkinson's disease (PD). ${ }^{2}$ So far, more than 300 patients with PD have been treated with fetal mesencephalic tissue. Though the results have been variable, several centres have observed consistent and clinically meaningful benefits in open trials. To investigate further the efficacy of this new medical technique, double blind, placebo-controlled studies have recently been initiated. ${ }^{3}$ This research design is advocated as the only way to exclude the possibility that the apparent benefits of EFTT in patients with PD are due to a placebo effect or investigator bias.

The ethical aspects of new medical technologies involve two kinds of moral issues. ${ }^{5}$ Questions of application arise within the framework of the technology. They proceed from an acceptance of the technology and attempt to define responsible and appropriate use. They cover the medical, social, economic and ethical consequences of the introduction of a particular technology, and the fair distribution of that technology among possible beneficiaries. Fundamental questions focus on whether the technology as such is justified in the light of current moral norms and underlying values. From this perspective, elective abortions as a source of neural tissue raise fundamental moral problems and the debate will not easily cease. ${ }^{6}$ Recently, ethical guidelines specifically formulated for retrieval and use of fetal tissue for the purpose of experimental and clinical neurotransplantation have been published as self-restraining rules by the Network of European CNS Transplantation And Restoration (NECTAR). ${ }^{78}$ These guidelines remain under continuous debate in the European Community, which debate will not be discussed in this paper.

We focus on the first type of issue, specifically on the problem of whether it is morally acceptable to perform sham surgery in an experimental study in order to assess the efficacy of intracerebral transplantation of fetal nervous tissue in patients with PD. Neither the retrieval of tissue, nor the technique of EFTT as such is questioned, only the sham element. The recent debate in The New England Fournal of Medicine is reviewed. Subsequently, it is argued that a sham procedure is morally unacceptable. One of the arguments is that there is an alternative, less harmful, method to assess the efficacy of EFTT in patients with PD.

\section{Neural grafting in patients with PD}

A rapid development of neural grafting in patients with PD occurred after the observation that grafting fetal nigral cells into the striatum of substantia nigra-lesioned rats reversed the motor disturbances of these rats. Because the rat model only partially represents the complexity of the disease in humans, transplantation studies in non-human primates were also performed, which essentially validated the process in rats. Neurotransplantation in PD patients began with open trials of intracerebral placement of the patient's own adrenal medulla tissue. This tissue was known to be an alternative source of dopamine, and was chosen as an alternative to human fetal tissue, to circumvent the moral objections likely to be raised 
by the use of human fetal brain tissue obtained from elective abortions. These experimental studies largely failed, however, and a move towards the use of human fetal mesencephalic tissue appeared to be inescapable. ${ }^{9}$

The first clinical studies with human fetal brain grafts were performed in 1987. Since then, over 300 patients worldwide have received this experimental treatment, and it has resulted in persistent and significant but none the less incomplete and variable alleviation of the disease symptoms. In practice, neurotransplantation in PD patients takes the form of a precisely directed injection of $\mu l$ quantities of a suspension of fetal nerve cells or tissue fragments $\left(10^{-6}\right.$ to $\left.10^{-5} \mathrm{l}\right)$ prepared from specific areas of the embryonic or fetal brain. This type of surgery requires precision and accuracy, but does not constitute an extremely severe, physically invasive operation on the patient. The potential therapeutic benefits of the transplantation of fetal brain tissue are therefore not immediately apparent, but develop over a period of several months, not unlike brain cell maturation in the intrauterine fetal stages.

\section{The current debate}

The problem of the assessment of the efficacy of a surgical procedure has been pointedly described by Johnson:

"There is an aura around the whole process of a surgical operation: the expectations, the surroundings, the surgeon's personality, the anaesthetic, and the incision all contribute to the outcome, quite apart from the specific effect of the surgical procedure. Expectations are influenced by the experiences of friends and of other patients, the length of the illness, the amount of pain, and accounts of surgery on television or in magazines. Assessment of the outcome can also be influenced by the hopes and pride of both the surgeon and the patient. . . New technical advances must be assessed carefully. For surgical procedures, as for drugs, the placebo effect must always be taken into account if any assessment is to be objective." 10

Though there is a growing interest in alternatives (such as $\mathrm{n}=1$ trials), for about four decades, randomised, double blind, placebo-controlled trials (RCT's) have been the gold standard in biomedical research. They are generally considered the most satisfactory research design in evidence-based medicine. Should they be used in the assessment of the efficacy of neural grafting in patients with PD? The ethical debate recently appeared in The New England Fournal of Medicine. A plea in favour of sham surgery by workers in the field has been criticised by a bioethicist in what is essentially a plea against sham surgery.

A PLEA IN FAVOUR

A plea in favour was presented by Freeman $e t a l .^{3}$ In their study, 24 patients underwent neural grafting and 12 patients received sham surgery. The sham procedure includes the placement of a stereotactic frame, target localisation on magnetic resonance imaging, anaesthesia, and a skin incision with a partial burr hole, but no needle penetration into the brain and no implantation of fetal brain tissue. The central question of Freeman et al is: "Under what circumstances are the risks to subjects assigned to the placebo group in a medical or surgical trial justified, and what risks are reasonable in order to determine the benefits and adverse effects of a given intervention?" 11 This quotation concerns a question of a particular application of EFTT, since sham surgery as such is not debated.

Freeman et al list three criteria that must be met before randomised, double blind, placebocontrolled trials can be carried out: (1) they should address an important research question that cannot be answered by a study with an alternative design, which poses a lower risk to the subjects; (2) there must be preliminary but not conclusive evidence that the intervention is effective, and (3) the treatment should be developed to the point where it is unlikely to become obsolete before the study has been completed.

Though the results of the implantation of fetal mesencephalic tissue in the striatum of patients with PD have been variable, several centres have observed consistent and clinically meaningful benefits in open trials (criterion 2). In scientific reports this technique is considered a promising intervention, where neuronal cell survival needs to be improved by further study and experimentation (criterion 3). ${ }^{12}{ }^{13}$ The practical and moral difficulties of obtaining sufficient human fetal brain tissue have, meanwhile, encouraged studies of alternative sources, such as the use of xenografts from pig fetuses and the use of genetically modified or laboratory-cultured (non-)neuronal cells. The treatment of patients with PD with fetal mesencephalic tissue is still open to further research, but the question arises whether one needs to use a placebo control rather than some alternative design (without a placebo control) to reduce the risk to the research subjects. The most problematic criterion of Freeman et al is, therefore, the first one.

Freeman et al, however, conclude (1) that the risks of participating in the study are "reasonable" in relation to the possible benefits, and (2) that it is necessary to perform a double blind, placebocontrolled study, because this is the only way to exclude the possibility that the benefits are due to a placebo effect or investigator bias.

\section{A PLEA AGAINST}

For Macklin, a bioethicist asked to comment on the publication of Freeman et al, the central question is: "Why should the use of sham surgery be questioned when the conditions for the ethical acceptability of a placebo-controlled study appear to be met?" ${ }^{14}$ Her considerations involve a fundamental moral evaluation of sham surgery. Macklin focuses on three critical points: (1) the tension between the highest standard of research design and the highest standard of ethics, (2) the problem of how to 
proceed with the ongoing uncertainties and disagreements in assessing the risks and benefits of research protocols, and (3) the relationship between the risks of a protocol and the informed consent of research subjects.

On the first point, it is remarkable that Macklin contrasts the highest standard of research design with the highest standard of ethics. This denies that research ethics takes into account not only the interests of research subjects, but also the interests of biomedical science, of the category of patients to which the research subjects belong, and of society at large. However, Macklin does seem to accept that ethical aspects also involve the scientific methodology, such as the randomisation procedure and the use of placebos. Thus, one could better speak of a tension between the interests of the research subject, and the interests of the researcher, of science, and of society, and compare the quality of the research design with the quality of the research subjects' interest.

Regarding the second point, Macklin argues that research ethics committees can run into considerable difficulties in making reliable and consisten assessments of the benefits and risks of a research protocol. Studies of sham surgery in patients with PD have generated a variety of reactions, amounting to a lack of consensus. A lack of consensus is a strong impetus for continuing the bioethical debate. Macklin sees this as an argument against the use of sham surgery.

Finally, Macklin argues that the disclosure of the research design does not eliminate the known placebo effect of surgery, and that the informed consent of the participant is not the only ethical requirement for conducting research. Subsequently, she asks the crucial question: "Is it overly paternalistic to protect research subjects from risks they seem willing to accept?" Her answer is "no" and she adds that the emphasis today on respect for the autonomy of research subjects makes researchers reluctant to ask whether their choices are fully rational.

Macklin concludes (1) that the protection of human subjects cannot rest solely on the ethical foundation of informed consent; (2) that it cannot be maintained that no deception was involved in the use of sham surgery, and (3) that sham surgery is ethically unacceptable as a placebo control in trials of fetal brain cell transplantation in patients with PD.

\section{Discussion}

Freeman et al argue that animal models for Parkinson's disease are of limited value because they never completely match the symptomatology and prognoses of the human condition. Thus the assessment of the final efficacy of any neurotransplantation approach will require clinical trials, with some risk of negative effects in human beings.

One of the arguments in favour of sham surgery, according to Freeman et al, is based on the distinction between (1) pharmaceutical interventions, (2) traditional or conventional surgery, and (3) surgical procedures for cell implantation. The authors argue that cell therapies have more in common with pharmaceutical interventions than with conventional surgery. However, cell therapies as discussed here (intracerebral injections after drilling a hole in the skull) involve a more invasive and irreversible procedure than do pharmaceutical interventions. Therefore, cell therapies - and definitely intracerebral cellular therapy-should be seen as much more akin to traditional surgery. All the (false) expectations mentioned in the above quotation from Johnson are then applicable to EFTT, and the need for an adequate control assessment becomes obvious. It is, however, questionable whether the risks of sham surgery are as "reasonable" in relation to the possible benefits, as Freeman et al argue (see below).

Another problematic point in the study of Freeman et al is that it is not a "real" placebo intervention. Their study was not designed to check for any clinical change due to the surgical lesion itself, since this would entail substantial additional risk for the control group. Moreover, existing data would suggest that surgical trauma or implantation of biologically inactive tissue does not result in significant improvement in primate models of parkinsonism. ${ }^{3}$ However, this result from research with primates does not mean that a placebo effect of the surgical procedure itself can be excluded in patients. A "real" placebo intervention would include a penetration of the inner cortex of the skull and a needle penetration into the recipient areas of the brain. Instead of (possibly) effective fetal grafts, biologically ineffective fetal tissue or at least a volume of saline would then be injected. Thus, the study design of Freeman et al offers no or only partial control for the bias effect of the surgical procedure in PD patients.

Macklin raises another important point to challenge sham surgery. Informed consent is not the only ethical standard to be met for ethically conducted scientific research with human beings. Sham surgery involves active deception in one way or another, that can give rise to unwanted side effects of a psychological nature (see below).

\section{The need for sham surgery challenged}

Summarising, sham neurosurgery in patients with PD raises pressing methodological and ethical problems. As Freeman et al suggest (their first criterion), a key question is whether there is an alternative, less harmful, research design. We will address this question below. But apart from the existence of an alternative research design there are more fundamental arguments (as advanced by Macklin) that make it undesirable to accept sham surgery in patients with PD. If there was no alternative research design one could still challenge the moral acceptability of sham surgery. In this context, the principles of respect for autonomy (and the related notion of therapeutic misconception), of the integrity of the human body, of beneficence and of non-maleficence play a crucial role. 
RESPECT FOR AUTONOMY

The first requirement (noted in the Nuremberg Code of 1947) for ethical research is the autonomous, voluntary, informed consent of the research subjects. Through informed consent the investigator and the subject enter into a fiduciary relationship which requires reciprocal respect and communication. According to Levine, the most appropriate single word for the communication between researcher and research subject is "negotiation". ${ }^{15}$ The negotiations must include the exchange of information, the assessment of the prospective consentor's comprehension, the assessment of the prospective consentor's autonomy, and giving consent. Sham neurosurgery is clearly research bearing more than minimal risk. Thus, there must be a great deal of negotiation between researchers and prospective research subjects. Because in the study being considered the research subjects are patients with PD, it is unlikely that there will be problems in the assessment of their comprehension of the study, or in the assessment of their autonomy. Patients with PD selected for brain tissue grafting are generally regarded as competent to give consent. ${ }^{8}$ A potential problem arises when it comes to recruiting patients. How can one be sure that they view themselves as absolutely free to choose whether or not to participate in a research project? And how to avoid "therapeutic misconception"?

\section{THERAPEUTIC MISCONCEPTION}

The notion of therapeutic misconception encompasses the idea that clinical research using patients as subjects and directed toward developing treatment, offers the potential for direct benefit to patients. Many patients not only agree to participate in studies such as these, but also eagerly desire participation, because they believe, often erroneously, that the study represents benefits to them. The expectation of research subjects may be unrealistic. In consequence, it is likely that the consent will not really be freely given. Thus, an unrealistic expectation of benefit undermines the validity of the consent given by the patient. Then, the principle of informed consent and that of free consent are both corrupted. In this context it is striking that in a study of fetal tissue transplantation in patients with PD, one of the participants and his wife felt "double shammed". ${ }^{16}$ First, they felt betrayed when they learned that he had undergone the sham procedure (although the consent form did state that this could happen). Second, the patient was disappointed, when he was denied the real surgery after the study on the basis of safety considerations. In any case, it is morally debatable to promise on the consent form a treatment of not yet known benefit (that is, the active cell implantation) after completing the study. But reactions such as that of the patient just mentioned are an additional reason to be very cautious in this matter. There can be confusion and betrayal of trust when patients, afterwards, realise that the optimistic assessment of their chance of benefit was really a misconception.
Is this a sufficient reason to protect research subjects from taking part in the study, while they still hope for a direct benefit? According to Macklin, it is not overly paternalistic to try to prevent the patient from participating in studies like these.

INTEGRITY OF THE HUMAN BODY

Apart from the requirement for a patient's informed consent, the question arises whether drilling a hole in the skull and subjecting the patient to other medical regimes in a sham procedure (thus, with a risk of harm and without possible benefits to the research subject) are morally acceptable. Questions such as this presuppose that the human body is not just an instrument of the person, and that the "owner" of the body has no absolute right to (mis)use it. The integrity of the human body implies, that although research subjects have given explicit consent to a particular manipulation of their body, one must still ask, as Macklin rightly does, whether that manipulation is morally acceptable. This is not to say that the integrity of the human body is an absolute argument against sham surgery. One can say that drilling two holes in the outer layer of the skull is no serious violation of the body's integrity. There are people who wear-as an alternative to tattoos and piercings-two horns which are fixed in holes in their frontal skull. Moreover, one can say that, while EFTT in patients with PD is generally regarded as a promising development, the possible benefits of EFTT for future patients may counterbalance the risks associated with the placebo procedure. Thus, sham surgery in neurotransplantation must not unreflectedly be excluded, but the integrity of the human body must always be taken into account.

\section{BENEFICENCE AND MALEFICENCE}

The most difficult question to answer, is whether there is a reasonable "balance" between the clinical and scientific value of a particular study, and the burden it places on research subjects. The discussion in The New England Fournal of Medicine concentrates rather heavily on the risks to research subjects. The ethical debate can be extended by explicitly focusing on the possible benefits of the research protocol, especially on the distinction between direct, collateral and aspirational benefit. ${ }^{17}$ Direct benefit to subjects arises from receiving the intervention being studied. Collateral benefit arises from being a research subject, even if one does not receive the experimental intervention: for example, a free physical exam, free medical care, and - in the study being discussed-the possibility of receiving fetal tissue after completing the study in the placebo group, if EFTT is found to be effective. Aspirational benefit is the benefit to society and to future patients arising from the subjects' altruism and the results of the study.

In clinical research designed to develop future treatments, it is the possibility of direct benefit that is of greatest interest to patient-subjects. But due to (potential) conflicts of interests ${ }^{18}$ it is quite common 
to conflate direct benefit with collateral and aspirational benefits. Therefore, an ethical review of sham surgery of patients with PD must focus on a weighting process at an inter-individual level, because it is unknown whether the research subjects will benefit from the study. The burden for them must be weighed against the possible benefits for future patients with PD. While it is relatively easy to assess the burden and benefits for the research subjects here and now, however, it is far more difficult to weigh the possible benefits for all future patients with PD. The more so since the current double blind studies are performed with a grafting technique that still requires development to improve the survival rate of the implanted cells.

Another question that arises is: what are in fact the "clinically meaningful benefits" of EFTT in PD patients? How long does fetal transplant function in patients with PD? Does the intervention promise a complete and permanent cure, or is it just a temporary or partial improvement while the patient remains disabled? Freeman et al end their article as follows: "If fetal tissue transplants are found to be safe and effective, thousands of patients with Parkinson's disease stand to benefit, and further research will be encouraged. If the transplants are found to be unsafe or ineffective, or if they offer nothing more than a placebo effect, hundreds or even thousands of patients will be spared the risks and financial burdens of an unproved operation."19 At first sight, this seems to be a strong argument in favour of sham surgery. However, the safety of the procedure is no longer in doubt, and an alternative research design to assess the efficacy (see below) does not require the involvement of hundreds of patients. The argument also bypasses the central problem: how to assess the relation between the clinical and scientific value of a particular study and the burden it may cause to research subjects. As Macklin argues, in trials involving sham surgery in patients with PD, the risk-benefit-ratio is at best uncertain and at worst unfavourable.

CAP RATHER THAN SHAM

A final argument against sham surgery is that there is an alternative research design that can produce the same or comparable scientific evidence. Controlling the effectiveness of fetal tissue neurotransplantation in PD implies assessment at two levels at least. First, do human fetal cells of the graft survive and integrate with the host brain to provide renewed dopaminergic input for the enervated areas? Second, does the surviving dopamineproducing graft ameliorate the disease symptoms? Animal research with PD models gave a positive answer to both questions. However, the rat and monkey disease models are all acute lesion models, and therefore do not match the long term, slow degeneration of human PD. ${ }^{9}$ Thus, patient studies were necessary. Most of these studies have been performed as open trials, but some groups introduced a scheme of pre- and post-surgery evaluation of the parkinsonian symptoms to control for the patient intra and intervariability and thus permit the effectiveness of the treatment to be monitored in greater detail.

Concerning the assessment of the efficacy of human fetal mesencephalic tissue grafting, one can safely say there is no necessity to establish tissue survival with control surgery: (1) Positron Emission Tomography (PET) imaging of critical markers has shown that human fetal dopaminergic neurons survive grafting ${ }^{20} ;(2)$ postmortem histological studies of patients who have died long after implantation surgery, have shown extensive cellular integration to the same degree as seen in animal experiments ${ }^{21}$; (3) in vivo release of dopamine from the transplant has recently been reported. ${ }^{22}$ These are all quantifiable data, some of which can be measured before and after neurotransplantation surgery, ie, in an intrapatient study design.

Quantitative evaluation of the amelioration of the disease symptoms of the patient is more difficult since PD rating scales are largely subjective. Daily fluctuations make scientific evaluation even more difficult, as does the inclusion of the patient's own self-scoring data. The introduction of a diseasededicated core assessment protocol (CAP) has helped to overcome these problems. ${ }^{23}{ }^{24}$ With this protocol a stable presurgery state is determined and the same metric is applied postsurgery on a strict time schedule for a period long enough to cover the time required for the grafted neurons to mature and become functional. However, a CAP cannot entirely eliminate observer and patient bias about the outcome of the neurotransplantation surgery. If quantifiable measures specifically related to disease symptoms are incorporated in the CAP, and "control" patients maintained on their standard treatment are randomly incorporated and subjected to the same CAP, scientifically unbiased observations (or at least observations with a minimal bias) can be obtained. Then, there is no need to perform control surgery to establish the functional significance of the transplant. The development of specific CAPs rather than sham surgery should thus be advocated.

\section{Conclusion}

Though the results of neurotransplantation in patients with $\mathrm{PD}$ have been variable, this technique is considered a promising intervention which needs improvement. While the surgical procedure is a physically invasive (though not severe) operation, it remains unclear how far the benefits of this experimental technique are due to a placebo effect or investigator bias. This state of uncertainty in the clinical community about the preferred treatment has been called "clinical equipoise". ${ }^{25}$ Thus, from a strict methodological point of view, randomised, double blind, placebo-controlled studies are indicated. However, this methodological ideal raises pressing ethical problems. An ideal placebo procedure must fulfil the criteria of (1) being supposedly ineffective, (2) having no (serious) side effects, and (3) being a true copy of the intervention under study. We argued that such a placebo procedure does not (yet) exist and is also not morally justified in view of the present state of the art. 
We advanced a number of arguments as a plea against sham surgery in neurotransplantation research in PD patients. The notions of therapeutic misconception and of the integrity of the body, and the difficulties in assessing the balance between risks and benefits provide strong arguments against sham surgery, but are in themselves not decisive. The above arguments do not mean that the sham procedure in patients with PD as such is unethical and that sham surgery in neurotransplantation is $a$ priori excluded. The risk of harm without possible benefit is in itself not a valid argument against sham surgery. It must be weighed against the category of PD patients' interests and the existence of an alternative research design.

Therefore, a valid additional and decisive argument against sham surgery (to which too little attention has been paid in the discussion in The New England Fournal of Medicine) is the existence of an alternative, less harmful research design that can provide the same or comparable empirical evidence. We believe that intrapatient research is a superior alternative. Though this design does not offer the validity of an RCT, it can provide comparable empirical evidence. Our design requires the application of a core assessment protocol (CAP), based on quantitative measures, on a rigid schedule running from the pre-operative period through to postconvalescence. This guarantees that objective data are collected (pre and postoperative), that can be compared with a parallel CAP-evaluated randomly assigned reference group of patients who have not been subjected to any surgical intervention.

Finally, the focus in this paper is on neurosurgery in patients with $\mathrm{PD}$, and especially on nigral cell implantations, but our arguments are equally applicable to a wider therapeutic arsenal. From a methodological point of view, other experimental surgical interventions in PD such as putative neurosurgical therapies, strategic lesions in the outflow pathways of the basal ganglia, and the placement of electrical cerebral stimulators would also require sham interventions to assess their efficacy. Observer and patient bias can then similarly be expected. Here a validated PD-specific CAP would also be of value as an evaluation instrument.

\section{Acknowledgements}

This paper developed from discussions at the third meeting of the BIOMED project, Ethical guidance on embryonic and fetal tissue transplantation, Barcelona, Spain, January $18-19,2000$. It was financed by the European Committee under no BMH4CT98-3928 (DG12-SSMI). We would like to thank Gerhard Zielhuis for his comments on an earlier version of this paper.

Wim Dekkers, MD, PhD, is Senior Lecturer in Medical Philosophy, Department of Ethics, Philosophy and History of Medicine, University Medical Centre, Nijmegen and Senior Researcher, Centre for Ethics, University of Nijmegen, the Netherlands. Gerard Boer, PhD, is Senior Researcher, Netherlands Institute for Brain Research, Amsterdam, the Netherlands.

\section{References}

1 Dekkers WJM, Have HAMJ ten. Biomedical research with human body parts. In: Have HAMJ ten, Welie JVM, eds. Ownership of the human body. Dordrecht: Kluwer Academic Publishership of the human

2 Lindvall O. Cerebral implantation in movement disorders: state of the art. Movement Disorders 1999;14:201-5.

3 Freeman ThB, Vawter DE, Leaverton PE, Godbold JH, 3 Freeman ThB, Vawter DE, Leaverton PE, Godbold JH, led trials of a cellular-based therapy for Parkinson's disease. The New England fournal of Medicine 1999;341:988-91.

4 Fahn S, Greene PE, Tsai W-Y, Eidelberg D, Winfield H, Dillon $\mathrm{S}$, et al. Double-blind controlled trial of human embryonic dopaminergic tissue transplants in advanced Parkinson's disease [abstract]. Neurology 1999; 52(suppl 2):A405.

5 Have HAMJ ten. Medical technology assessment and ethics. Hastings Center Report 1995;25:13-9.

6 Keown J. The Polkinghorne report on fetal research: nice recommendations, shame about the reasoning. Fournal of Medical Ethics 1993;19:114-20.

7 Boer GJ. Ethical guidelines for the use of human embryonic or fetal tissue for experimental and clinical neurotransplantation fetal tissue for experimental and clinical neurotra

8 Boer GJ. The self-restraining ethical guidelines of NECTAR for the clinical neurotransplantation investigations. In: Hubig C, Poser C, eds. Cognitio humana-Dynamik des Wissens und der Werte. Leipzig: XVII Deutscher Kongress für Philosophie, 1996:1420-7

9 Boer GJ. Ethical issues in neurografting of human embryonic cells. Theoretical Medicine and Bioethics 1999;20:461-75.

10 Johnson AG. Surgery as placebo. Lancet. 1994;344:1140-2.

11 See reference 3: 988 .

12 Brundin P, Karlsson J, Emgård M, Kaminski Schierle GS, Hansson O, Petersen A, et al. Improving the survival of grafted dopaminergic neurons: a review of current approaches. Cell Transplantation 2000;9:179-95.

13 Health Council of the Netherlands, Standing Committee on Medicine and Standing Committee on Medical Ethics \& Mealth Law. Fetal tissue transplantation. Rijswijk: Health CounHealth Law. Fetal tissue transpla
cil of the Netherlands, 1997.

14 Macklin R. The ethical problems with sham surgery in clinical research. The New England fournal of Medicine 1999;341:992-6.

15 Levine RJ. Ethics and regulation of clinical research. New Haven/ London: Yale University Press, 1986: 99.

16 See reference 14: 994

17 King NMP. Defining and describing benefit appropriately in clinical trials. Fournal of Law, Medicine, E Ethics, 2001;28:33243.

18 Little M. Research, ethics and conflicts of interest. Fournal of Medical Ethics 1999;25:259-62.

9 See reference 3: 991.

20 Peschanski M, Defer G, N'Guyen JP, Ricolfi F, Monfort JC, Remy P, et al. Bilateral motor improvement and alteration of L-dopa effect in two patients with Parkinson's disease following intrastriatal transplantation of foetal ventral mesencephalon. Brain 1994;117:487-99.

21 Olanow CW, Kordower JH, Freeman TB. Fetal nigral transplantation as a therapy for Parkinson's disease. Trends in Neuroscience 1996;19:102-9.

22 Piccini P, Brooks DJ, Björklund A, Gunn RN, Grasby PM, Rimoldi $\mathrm{O}$, et al. Dopaminergic release from nigral transplants visualized in vivo in a Parkinson's patient. Nature Neuroscience 1999;2:1137-40.

23 Langston JW, Widner H, Goetz CG, Brooks D, Fahn S, Freeman $\mathrm{T}$, et al. Core assessment program for intracerebral transplantation (CAPIT). Movement Disorders 1992;7:2-13.

24 Widner H, Defer G-L. Dyskinesias assessment: from CAPIT to CAPSIT. Movement Disorders 1999;14(suppl 10):60-6.

25 Freedman B. Equipoise and the ethics of clinical research. The New England fournal of Medicine 1987;317:141-5. 